\title{
Causas atribuidas a eventos de aparente amenaza a la vida del lactante
}

\author{
DANIEL ZENTENO A. ${ }^{1}$, GONZALO QUIROZ Z. ${ }^{2}$, MAGDALENA CELIS B. ${ }^{3}$, JAIME TAPIA Z. ${ }^{3}$
}

1. Médico Pediatra, Becado de Neumología, Departamento de Pediatría Sur, Facultad de Medicina, Universidad de Chile.

2. Interno de Medicina, Facultad de Medicina, Universidad de Concepción.

3. Médico Pediatra, Departamento de Pediatría, Facultad de Medicina Universidad de Concepción. Servicio de Pediatría, Hospital Guillermo Grant Benavente.

\begin{abstract}
Causes attributed to apparent life-threatening event: 3 years of experience

Background: An Apparent Life-Threatening Event (ALTE) is an important challenge for the pediatrician, which requires guidelines for finding the etiology and making appropriate clinical decisions. Objective: To describe etiologies and clinical features of ALTE in infants, as determined by a standarized protocol. Methods: A prospective study including infants admitted to the Emergency Room and hospitalized due to an ALTE between May 2002 and May 2005. They were evaluated with an established protocol that considers the episode description, clinical history, and complementary laboratory studies. Results: 109 patients with ALTE and a mean age of 11 weeks had the following symptoms: apnea (100\%), skin color changes (97\%), and muscle tone changes (83\%). 96\% received CPR maneuvers or strong stimulation maneuvers for ending the episode. Fourteen percent of the cases were idiopathic, while other etiologies included respiratory infections (46\%), gastroesophageal reflux (16\%), seizures (6\%), and urinary tract infections (6\%). 3 patients expired. Conclusions: With this protocol, based on tools available in most public tertiary hospitals in Chile, we can identify the causes of ALTE similar to those reported in other studies, howeber with a lesser percentage of idiopathic episodes.
\end{abstract}

(Key words: Apparent Life-Threatening Event, apnea, infants).

Rev Chil Pediatr 2008; 79 (2): 163-171

\section{RESUMEN}

Introducción: Un Evento de Aparente Amenaza a la Vida (ALTE) plantea un importante desafío al pediatra, quién debe investigar la etiología para orientar su conducta. Esta labor se facilita con la aplicación de una guía. Objetivo: Describir la presentación clínica y determinar posibles causas de ALTE mediante un protocolo especifico. Pacientes y Método: Estudio prospectivo en lactantes que consultaron en un 
Servicio de Urgencia y se hospitalizaron por ALTE entre Mayo 2002 a Mayo 2005. Se evaluaron con un protocolo preestablecido considerando la descripción del episodio, antecedentes y estudios complementarios por pasos. Resultados: En 109 pacientes con ALTE, edad promedio 11 semanas, se observó apnea en $100 \%$ y cambio de color en $97 \%$. El $83 \%$ presentó cambio de tono y en el 96\% se utilizaron maniobras para abortar el episodio. Se consideró idiopático el $14 \%$ de los casos. Las principales causas atribuidas fueron: infecciones respiratorias (46\%), reflujo gastroesofágico (16\%), convulsiones (6\%) e infecciones urinarias (6\%). Fallecieron 3 pacientes. Conclusiones: Mediante este protocolo, que utiliza herramientas disponibles en la mayoría de los hospitales públicos terciarios de nuestro país, se atribuyeron causas similares a las reportadas en otros estudios prospectivos, donde destaca un menor porcentaje de episodios idiopáticos.

(Palabras clave: Eventos de Aparente Amenaza a la Vida, Apnea, lactantes).

Rev Chil Pediatr 2008; 79 (2): 163-171

\section{Introducción}

Un evento de aparente amenaza a la vida, denominado en Inglés ALTE (Apparent Life Threatening Event), se define como un episodio inesperado y brusco que alarma al observador, caracterizado por uno o más de los siguientes signos; compromiso de la actividad respiratoria (apnea, ahogo o sofocación), cambio de coloración (cianosis, palidez o rubicundez) y/o alteraciones del tono muscular (hipo o hipertonía) ${ }^{1}$.

El ALTE no es una enfermedad específica en sí misma, sino una forma de presentación clínica de diversos problemas o patologías ${ }^{2}$.

Se presenta de preferencia en lactantes alrededor de los dos meses de vida, siendo su etiología variada. Hay casos en los cuales no es posible atribuir la causa a una patología determinada (idiopáticos) ${ }^{3}$. La incidencia de ALTE es muy variable según distintos estudios internacionales. Un estudio realizado en Nueva Zelanda informó que 9,4 de mil nacidos vivos ingresaron a hospitales a causa de $\mathrm{ALTE}^{4}$. En centros Franceses el 2\% de los niños internados en centros pediátricos corresponden a ALTE $^{2}$. En Chile, no existen estudios publicados sobre estos aspectos epidemiológicos ${ }^{5}$.

Los niños que consultan por un ALTE son, aún en la actualidad, un desafío para el médico, en especial en lo que se refiere a su enfoque etiológico, a pesar de los avances en los procedimientos de diagnóstico ${ }^{6}$.

Nuestro objetivo fue describir la presentación clínica y probables etiologías en lactantes ingresados con el diagnóstico de ALTE según un protocolo diseñado para su enfrentamiento clínico utilizado en el Servicio de Urgencia y la Unidad de Lactantes de Servicio de Pediatría del Hospital Guillermo Grant Benavente, Concepción, Chile.

\section{Pacientes y Métodos}

Se efectuó un estudio descriptivo prospectivo en todos los niños entre un mes y dos años de edad, ingresados por ALTE que consultaron en el Servicio de Urgencia y se hospitalizaron en la Unidad de Lactantes del Hospital Guillermo Grant Benavente, Concepción, Chile, en el período comprendido entre el 1 de Mayo de 2002 y 1 de Mayo de 2005. La Comisión de Ética del establecimiento aprobó el estudio y los padres firmaron consentimiento informado.

Se registraron en un documento ad-hoc antecedentes generales, demográficos, perinatales, mórbidos, familiares (Síndrome de Muerte Súbita del Lactante (SMSL), ALTE, asma o epilepsia), consumo de tabaco en el grupo familiar, de alcohol o drogas, ingestión de medicamentos en las 24 horas anteriores, hábitos del sueño (ronquido, colecho, posición para dormir) y técnica alimentaria. Además, se registraron datos del episodio que motivó la consulta al Servicio de Urgencia: apnea o pausa respiratoria, cambio de color, cambio de tono muscular, estado de sueño o vigilia, maniobra que se realizó para abortar el evento, relación con 
factores que podrían relacionarse con el evento (alimentación, posición, llanto, fiebre, reflujo y tos) y personas presentes en ese momento. Se constató el examen físico completo: estado general, conciencia, signos vitales, saturación de oxígeno, color, tono, esfuerzo respiratorio, examen segmentario y desarrollo psicomotor.

Se realizó estudio con exámenes complementarios por etapas en aquellos lactantes cuya causa no era atribuible mediante la anamnesis y examen físico, el que se estableció luego de una coordinación entre subespecialistas y médicos encargados del estudio, conforme a los recursos disponibles en nuestro hospital.

Es considerado por etapas, pues al determinar la causa en las fases iniciales, no se continúa con los pasos siguientes.

Paso I: Solicitado en el Servicio de Urgencia: Hemograma, glicemia, electrolitos plasmáticos, gases venosos, calcemia, fosfemia, Proteína C Reactiva (PCR), creatininemia, ácido láctico, amonemia, sedimento de orina y radiografía de tórax.

Paso II: Solicitado al primer día de hospitalización: Panel respiratorio (Virus Sincicial Respiratorio [VRS], Adenovirus, Influenza y Parainfluenza), inmunofluorescencia directa para Bortedella Pertussis, estudio de reflujo gastroesofágico (radioisotópico) y tamizaje metabólico en orina. Evaluaciones por: Otorrinolaringología, Neurología (con electroencefalograma de sueño) y Cardiología (con electrocardiograma).

Paso III: Solicitado en pacientes sin causas atribuibles hasta ese momento $\mathrm{y}$ en base a reuniones caso a caso. Hormonas tiroideas, carnitina en orina, estudios específicos solicitados por las subespecialidades mencionadas previamente (ecocardiografía, Tomografía axial computarizada o Resonancia nuclear magnética), Polisomnografía y Evaluaciones por otras subespecialidades (figura 1).

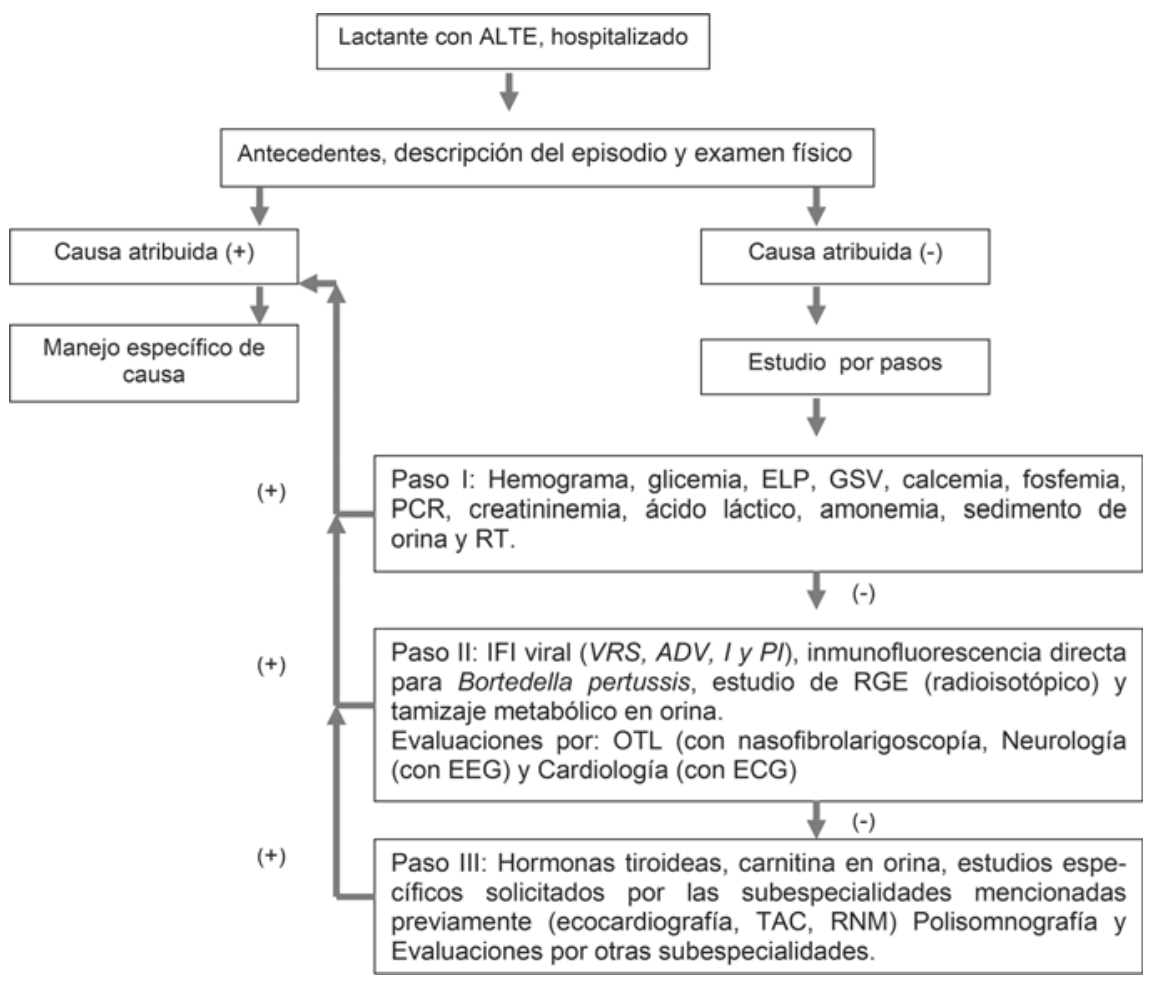

Figura 1. Algoritmo de estudio. ELP: electrolitos plasmáticos. GSV: gases en sangre venosa. PCR: proteína C reactiva. RT: Radiografía de tórax. IFI: Inmunofluorescencia indirecta. VRS: virus sincicial respiratorio. ADV: adenovirus. I: influenza. PI: parainfluenza. IFD: inmunofluorescencia directa. RGE: reflujo gastroesofágico. OTL: Otorrino. EEG: electroencefalograma. ECG: electrocardiograma. TAC: Tomografía axial computarizada. RNM: Resonancia nuclear magnética. 


\section{Resultados}

Entre el $1^{\circ}$ de Mayo del 2002 y el $1^{\circ}$ de mayo del 2005 se hospitalizaron 109 lactantes con diagnóstico de ALTE, los que fueron ingresados al estudio.

La edad promedio fue $11,3 \pm 9$ semanas (4 a 48 ), el $58 \%$ fue de sexo masculino, edad gestacional $37 \pm 4$ semanas (24-41), con un $31 \%$ de prematuros y $10 \%$ de bajo peso al nacer.

Los antecedentes familiares y del paciente se detallan en tabla 1.

El $100 \%$ de los casos presentó al ingreso apnea o pausa respiratoria, con una duración promedio de 0,81 minutos (0,2-4), el 14\% presentó apnea en más de una ocasión. Se observó cambio de coloración en el 97\% de los casos, 80\% de ellos correspondió a cianosis, y se realizaron maniobras de reanimación en el 97\%, siendo la estimulación vigorosa la más frecuente $(83 \%)$.

Las maniobras fueron practicadas por personas sin relación al área de la salud en el 90\% de los casos. La recuperación fue total en todos los pacientes.

Tabla 1. Antecedentes de los familiares, maternos y de los lactantes estudiados

\begin{tabular}{lcc}
\hline & $\begin{array}{c}\mathbf{n} \\
\text { de casos }\end{array}$ & $\%$ \\
\hline $\begin{array}{l}\text { Antecedentes maternos } \\
\text { Tabaquismo }\end{array}$ & 19 & \\
Alcohol & 9 & 17,4 \\
$\quad$ Drogas & 4 & 8,3 \\
Antecedentes familiares & & 3,7 \\
SMSL & 1 & \\
ALTE & 3 & 0,9 \\
Asma & 8 & 2,75 \\
Habitos del sueño & & 7,3 \\
Colecho & 75 & \\
Cohabitacion & 66 & 68,8 \\
Ronquido & 19 & 60,6 \\
Diaforesis & 17,4 \\
Pausas resp & 19 & 17,4 \\
Posición al dormir & 20 & 18,3 \\
Supino & & \\
Prono \\
Lateral
\end{tabular}

n: número
El 83\% de los pacientes presentó alteraciones del tono muscular, hipotonía en el $61 \%$ de ellos.

La tabla 2 muestra las características del episodio que motivaron el ingreso hospitalario.

El 68\% de los casos presentaban examen físico normal, con alteraciones en el 32\%, orientando en la mayoría a la etiología específica del ALTE.

Se estimó que 55\% requerían estudio complementario, el resto (45\%) bastó la anamnesis dirigida y examen físico para atribuir la causa. (tablas 3, 4 y 5).

Se consideró ALTE idiopático el 14\% y secundario $86 \%$.

Sobre la etiología atribuida por sistemas de los ALTE secundarios encontramos causas: Respiratorias 46\%, gastroenterológica 22\%, neurológica $11 \%$, urinaria $6 \%$, metabólica $0,9 \%$ y miscelánea $0,9 \%$

Tabla 2. Características del episodio de ALTE que motivo el ingreso hospitalario

\begin{tabular}{|c|c|c|}
\hline & $\begin{array}{c}n \\
\text { de casos }\end{array}$ & $\%$ \\
\hline Apnea o pausa respiratoria & 109 & 100 \\
\hline $\begin{array}{l}\text { Color } \\
\text { Cianosis } \\
\text { Cianosis central } \\
\text { Cianosis periférica } \\
\text { Palidez } \\
\text { Rubicundez } \\
\text { Normal }\end{array}$ & $\begin{array}{r}88 \\
44 \\
44 \\
16 \\
2 \\
3\end{array}$ & $\begin{array}{r}80 \\
40 \\
40 \\
15 \\
2 \\
3\end{array}$ \\
\hline $\begin{array}{l}\text { Tono } \\
\text { Hipotónico } \\
\text { Hipertónico } \\
\text { Normal }\end{array}$ & $\begin{array}{l}66 \\
24 \\
19\end{array}$ & $\begin{array}{l}60,5 \\
22 \\
17,4\end{array}$ \\
\hline $\begin{array}{l}\text { Reanimación } \\
\text { Si } \\
\text { No } \\
\text { Estimulación vigorosa } \\
\text { Boca a boca o nariz } \\
\text { Masaje cardíaco }\end{array}$ & $\begin{array}{r}104 \\
5 \\
90 \\
7 \\
7\end{array}$ & $\begin{array}{r}95,5 \\
4,5 \\
82,5 \\
6,5 \\
6,5\end{array}$ \\
\hline $\begin{array}{l}\text { Otros datos relacionados } \\
\text { Alimentación } \\
\text { Tos } \\
\text { Vómito } \\
\text { Regurgitación } \\
\text { Llanto } \\
\text { Cambio posición } \\
\text { Sobrecalentamiento } \\
\text { Fiebre } \\
\text { Movimiento asociado }\end{array}$ & $\begin{array}{r}21 \\
23 \\
8 \\
13 \\
13 \\
1 \\
2 \\
14 \\
7\end{array}$ & $\begin{array}{r}19,3 \\
21,1 \\
7,3 \\
11,9 \\
11,9 \\
0,9 \\
1,8 \\
12,8 \\
6,4\end{array}$ \\
\hline
\end{tabular}



das:

En cuanto a las causas específicas atribui-

Respiratorias; Principalmente por infección respiratoria aguda (IRA) baja, constituyendo un $26 \%$ del total de causas, luego IRA alta $21 \%$. De estas se consideraron de etiología viral el 74\%; VRS 9 casos, parainfluenza 2 e Influenza 1, mixtas 6 casos, 3 con VRS asociado, y bacterianas 7 casos, entre estas: Bortedella Pertussis 3, Chlamydia 1, Klebsiella
Pneumoniae 1, Staphylococcus Aureus, 1. En un paciente se diagnosticó fibrosis quística.

Gastroenterológica: Principalmente por RGE, $16 \%$, seguida por un $6 \%$ de casos atribuidos a errores en la técnica alimentaria.

Neurológica: En primer lugar por síndromes convulsivos $6 \%$, luego apnea emotiva $4 \%$.

Genitourinaria: 5\% por infección urinaria ITU), con urocultivos que demostraban presencia de Echerichia coli $>10^{5} / \mathrm{mm}^{3}$. En ninguno

Tabla 3. Causas generales y específicas de ALTE en todos los pacientes que integran el protocolo

\begin{tabular}{|c|c|c|c|c|c|}
\hline tipo & $\begin{array}{c}\text { Causas generales } \\
n^{\circ} \text { absoluto }\end{array}$ & $\%$ & tipo & $\begin{array}{l}\text { ausas específica } \\
n^{\circ} \text { absoluto }\end{array}$ & $\%$ \\
\hline Respiratoria & 50 & 45,8 & $\begin{array}{l}\text { IRAA } \\
\text { IRAB }\end{array}$ & $\begin{array}{l}21 \\
29\end{array}$ & $\begin{array}{l}19,2 \\
26,6\end{array}$ \\
\hline Gastrointestinal & 24 & 22,0 & $\begin{array}{l}\text { RGE } \\
\text { ETA }\end{array}$ & $\begin{array}{r}17 \\
7\end{array}$ & $\begin{array}{r}15,5 \\
6,4\end{array}$ \\
\hline Neurológica & 12 & 11,0 & $\begin{array}{l}\text { Sd convulsivo } \\
\text { Apnea emotiva } \\
\text { Conv febril } \\
\text { HIV }\end{array}$ & $\begin{array}{l}6 \\
4 \\
1 \\
1\end{array}$ & $\begin{array}{l}5,5 \\
3,6 \\
0,9 \\
0,9\end{array}$ \\
\hline Genitourinaria & 6 & 5,5 & ITU & 4 & 3,6 \\
\hline Metabólica & 1 & 0,9 & Galactosemia & 1 & 0,9 \\
\hline Otros & 1 & 0,9 & $\begin{array}{l}\text { Intoxicación } \\
\text { Farmacológica }\end{array}$ & 1 & 0,9 \\
\hline Idiopática & 12 & 11,0 & Idiopática & 12 & 11,0 \\
\hline Total & 109 & 100 & Total & 109 & 100 \\
\hline
\end{tabular}

IRAA: Infección Respiratoria Aguda Alta. IRAB: Infección Respiratoria Aguda Baja. RGE: Reflujo Gastroesofágico. ETA: Error Técnica alimentaria. ITU: Infección Tracto Urinario. HIV: Hemorragia intracraneana

Tabla 4. Causas generales y específicas de ALTE de los pacientes que sólo se utilizó anamenesis dirigida y examen físico

\begin{tabular}{|c|c|c|c|c|c|}
\hline tipo & $\begin{array}{c}\text { Causas generales } \\
n^{\circ} \text { absoluto }\end{array}$ & $\%$ & tipo & $\begin{array}{c}\text { ausas específica } \\
n^{\circ} \text { absoluto }\end{array}$ & $\%$ \\
\hline Respiratoria & 35 & 71,4 & $\begin{array}{l}\text { IRAA } \\
\text { IRAB }\end{array}$ & $\begin{array}{l}13 \\
22\end{array}$ & $\begin{array}{l}26,5 \\
44,8\end{array}$ \\
\hline Gastrointestinal & 7 & 14,2 & $\begin{array}{l}\text { RGE } \\
\text { ETA }\end{array}$ & $\begin{array}{l}2 \\
5\end{array}$ & $\begin{array}{r}4,0 \\
10,2\end{array}$ \\
\hline Neurológica & 3 & 6,1 & $\begin{array}{l}\text { Sd convulsivo } \\
\text { Apnea emotiva } \\
\text { HIV }\end{array}$ & $\begin{array}{l}1 \\
1 \\
1\end{array}$ & $\begin{array}{l}2,0 \\
2,0 \\
2,0\end{array}$ \\
\hline Genitourinaria & 3 & 6,1 & ITU & 3 & 6,1 \\
\hline Metabólica & - & - & & - & - \\
\hline Otros & 1 & 2,0 & $\begin{array}{l}\text { Intoxicación } \\
\text { Farmacológica }\end{array}$ & 1 & 2,0 \\
\hline
\end{tabular}

IRAA: Infección Respiratoria Aguda Alta. IRAB: Infección Respiratoria Aguda Baja. RGE: Reflujo Gastroesofágico. ETA: Error Técnica alimentaria. HIV: hemorragia intraventricular. ITU: Infección Tracto Urinario. HIV: Hemorragia intracraneana 
Tabla 5. Causas generales y específicas de ALTE en pacientes que se utilizó estudio complementario etapificado

\begin{tabular}{|c|c|c|c|c|c|}
\hline tipo & $\begin{array}{c}\text { Causas generales } \\
n^{\circ} \text { absoluto }\end{array}$ & $\%$ & tipo & $\begin{array}{c}\text { Causas específicas } \\
n^{\circ} \text { absoluto }\end{array}$ & $\%$ \\
\hline Respiratoria & 15 & 25,0 & $\begin{array}{l}\text { IRAA } \\
\text { IRAB }\end{array}$ & $\begin{array}{l}8 \\
7\end{array}$ & $\begin{array}{l}13,3 \\
11,6\end{array}$ \\
\hline Gastrointestinal & 17 & 28,3 & $\begin{array}{l}\text { RGE } \\
\text { ETA }\end{array}$ & $\begin{array}{r}15 \\
2\end{array}$ & $\begin{array}{r}25,0 \\
3,3\end{array}$ \\
\hline Neurológica & 9 & 15,0 & $\begin{array}{l}\text { Sd convulsivo } \\
\text { Apnea emotiva } \\
\text { Conv febril }\end{array}$ & $\begin{array}{l}5 \\
3 \\
1\end{array}$ & $\begin{array}{l}8,3 \\
5,0 \\
1,6\end{array}$ \\
\hline Genitourinaria & 3 & 5,0 & ITU & 3 & 5,0 \\
\hline Metabólica & 1 & 1,6 & Galactosemia & 1 & 1,6 \\
\hline Idiopática & 15 & 25,0 & Idiopática & 15 & 25,0 \\
\hline Total & 60 & 100 & Total & 60 & 100 \\
\hline
\end{tabular}

IRAA: Infección Respiratoria Aguda Alta. IRAB: Infección Respiratoria Aguda Baja. RGE: Reflujo Gastroesofágico. ETA: Error Técnica alimentaria. ITU: Infección Tracto Urinario.

se encontró alteraciones anatómicas del tracto genitourinario.

Metabólica: Un paciente con amonio y ácido láctico alterado que prosiguió estudio y se concluyó en una galactosemia clásica.

Miscelánea: 1 caso por intoxicación con fármaco antigripal compuesto.

El promedio de días de hospitalización fue 11,7 \pm 19 días (1 a 132). Las hospitalizaciones prolongadas fueron motivadas por el manejo complejo y/o evolución tórpida de causas secundarias específicas. Reingresaron el 8\% (9/109): 4 infecciones respiratorias (Síndrome Coqueluchoideo), 1 caso que presentaba ALTE severo en el cual finalmente se diagnósticó una fibrosis quística, 1 por RGE, 1 por síndrome convulsivo de difícil manejo, 1 ITU, y 1 causa idiopática.

Fallecieron 3 pacientes (3\%), 2 intrahospitalariamente. El primero ingresó por un ALTE secundario a bronconeumonia por virus parainfluenza con cardiopatía asociada no atribuible al ALTE, presentó sobreinfección bacteriana con Klebsiella pneumonia. Otro con antecedentes de Síndrome de Noonan y coartación aórtica operada que ingresó por bronconeumonia viral con germen no aislado, presentó ALTE recurrentes intrahospitalarios y evolucionó con sobreinfección por Staphylococcus Aureus. El último falleció extrahospitalariamente, 3 meses luego del alta con diagnóstico de ALTE secundario a bronconeumonia por VRS, no acudió a controles; su causa de muerte es discutible, pudiendo ser atribuida a muerte súbita o maltrato infantil.

\section{Discusión}

Los ALTE causan una gran ansiedad tanto en los padres como en las familias, quienes describen el episodio con una sensación inminente de muerte, exigiendo al clínico un conocimiento acabado acerca de la causa y evolución, lo cual no siempre es posible entregar en ese momento.

Nuestro estudio ha demostrado la utilidad del método estructurado que hemos implantado para ayudar al pediatra frente a un síndrome de etiología diversa y que muchas veces plantea dificultades de enfrentamiento. Con nuestro protocolo facilitamos la decisión al orientar hacia las etiologías más probables conforme a los medios que poseen gran parte de los hospitales terciarios en nuestro país, situación que explica la polisomnografía en la última etapa del protocolo.

Es preciso recalcar la subjetividad característica del ALTE, la información es entregada por padres o familiares en un contexto de angustia importante ${ }^{7}$. Nuestros casos fueron manejados inicialmente por personas no pertene- 
cientes al área de la Salud (90\%), quienes evaluaron y decidieron las medidas a tomar.

Samuels asegura que cuando es necesaria la reanimación cardiopulmonar (RCP) la mortalidad es alta $^{8}$. De nuestros casos, 2 niños fallecidos presentaban patologías graves después del episodio de ALTE y uno falleció de causas desconocida, probablemente por maltrato infantil.

A pesar de no contar con la tecnología de otros estudios publicados (registros fisiológicos, videos, monitoreo domiciliario etc), hemos logrado atribuir etiología en $86 \%$ de los casos. La tabla 6 muestra la comparación etiológica de nuestro estudio con otros publicados.

A diferencia de los otros estudios nuestros niños no fueron preseleccionados ni se excluyeron diagnósticos concomitantes. Davies eliminó a los mayores de 6 meses de vida y a los que presentaran fiebre ${ }^{3}$. Kahn excluyó a los niños que presentaban infecciones, anemia y perfil bioquímico alterado ${ }^{9}$, y Rahilly preseleccionó lactantes sin precisar el criterio ${ }^{10}$. Por estas consideraciones es difícil comparar nuestro trabajo con otros estudios de ALTE publicados. Algunos clínicos reservan su denominación solamente para los casos graves o recurrentes y excluyen a aquellos con causa determinada.

A pesar de todas estas limitaciones; la etiología atribuida de los casos de ALTE demostra- da en nuestro estudio es similar a la observada en las otras series publicadas. Al igual que otros estudios prospectivos, con protocolos estandarizados, se disminuye notablemente la etiología idiopática ${ }^{3}$.

En primer lugar hemos detectado las infecciones respiratorias, concordante con las estaciones del año en que ingresaron. Estas infecciones son consideradas como causales de ALTE y los expertos aconsejan practicar a todo niño con ALTE una radiografía de tórax a su ingreso, ya que en un estudio de ALTE, el 33\% los niños que presentaban infecciones respiratorias tenían el examen físico normal ${ }^{3}$. El $30 \%$ de nuestros lactantes con causa respiratoria atribuida tenía un examen físico normal e historia sin síntomas respiratorios.

En nuestro estudio, al igual que en otros estudios de nuestro medio ${ }^{5}$, los agentes más frecuentes fueron Virus Sincicial Respiratorio y Bortedella pertussis. Mc Carthy publicó una extensa revisión de la importancia de este virus en pediatría, el cual puede inducir apnea en un $25 \%$ de los niños hospitalizados por un mecanismo aun desconocido ${ }^{12}$. Rayyan determinó, mediante polisomnografía, que VRS podía causar apneas centrales antes de la aparición de síntomas respiratorios en lactantes menores $^{13}$. También Bortedella pertussis se asoció a ALTE, como en el estudio de Davies ${ }^{3}$ se ha comprobado que puede causar hipoxemia

Tabla 6. Etiología de ALTE: Comparación del estudio presente con investigaciones anteriores

\begin{tabular}{|c|c|c|c|c|c|}
\hline Estudio & $\mathbf{n}$ & Diseño estudio & Edad \% (s) & $\begin{array}{c}n \text { de } \\
\text { fallecidos }\end{array}$ & $\begin{array}{l}\text { Etiología de ALTE (\%). } \\
\text { Causas Principales }\end{array}$ \\
\hline Actual & 109 & Prospectivo & 11 & 3 & $\begin{array}{l}\text { Respiratorias (46), Gastroenterológica (22), } \\
\text { Idiopático (14), Neurológica (11), } \\
\text { Infección urinaria (6) }\end{array}$ \\
\hline Davies $^{3}$ & 65 & Prospectivo & 7 & 0 & $\begin{array}{l}\text { RGE (26), Idiopático(23), Pertussis (9), } \\
\text { Infección respiratoria (9), Convulsiones (9), } \\
\text { Infección urinaria (5) }\end{array}$ \\
\hline Kahn ${ }^{9}$ & 3799 & Prospectivo & 13 & $?$ & $\begin{array}{l}\text { Idiopático (38), RGE (20), Infección } \\
\text { respiratoria (7), Convulsiones (4) }\end{array}$ \\
\hline Rahilly ${ }^{10}$ & 340 & Prospectivo & 5 & 0 & Idiopático (15), RGE (62), Convulsiones (8) \\
\hline Veereman Wouters ${ }^{11}$ & 130 & Retrospectivo & Rango 2-36 & 2 & Idiopático (42), RGE (26), Convulsiones (4) \\
\hline Brockmann 5 & 71 & Retrospectivo & 9 & 0 & $\begin{array}{l}\text { Infección Respiratoria (29), Idiopático (36), } \\
\text { Síndrome convulsivo (12), Mala técnica } \\
\text { de alimentación (14) }\end{array}$ \\
\hline
\end{tabular}


severa en niños infectados ${ }^{14}$. En nuestros casos se detectaron otros agentes infecciosos como parainfluenza, en concordancia con los hallazgos de hipoxemia como se ha demostrado previamente ${ }^{15}$.

La segunda etiología atribuida fue el RGE y errores de técnica de alimentación. La relación entre el ALTE y el RGE ha sido ampliamente discutida, existiendo gran variabilidad entre los distintos estudios puesto que utilizan distintos criterios para determinar esta asociación. Actualmente la mayoría de los autores sugieren que para comprobar una relación con ALTE deberían realizarse estudios que demuestren una relación temporal entre ALTE y RGE, es decir una polisomnografía más Ph metría, que lamentablemente no esta disponible en la mayoría de los hospitales terciarios de nuestro país ${ }^{16}$. Wenzel demostró claramente una correlación entre la apnea y el RGE ${ }^{17}$. Mousa evidenció una relación temporal en el 15\% de los episodios de apnea observados, no influyendo el grado de acidez ${ }^{18}$. Nosotros, conforme a nuestros medios, utilizamos una anamnesis detallada en busca de RGE y una observación intrahospitalaria rigurosa; en casos de dudas nos apoyamos en signos indirectos de RGE detectados en la rinolaringoscopía y solicitamos el apoyo complementario con cintigrafía para RGE.

En cuanto a los errores de la técnica de alimentación, se han observado casos de ALTE durante el amamantamiento del lactante en posición incorrecta, o bien al sostenerlo en brazos de manera inadecuada ${ }^{19}$. Una buena anamnesis puede evitar hospitalizaciones y exámenes innecesarios en estos casos.

En tercer lugar, tal como en otros estudios prospectivos observamos apnea debida a crisis epilépticas lo cual demuestra la utilidad del EEG. Se recomienda este examen de rutina aun en ausencia de síntomas clínicos específicos pues el seguimiento de menores de un año que presentaron ALTE reveló una alta prevalencia de convulsiones $^{20}$.

Los episodios de apnea emotiva ocurren en un 3\% de lactantes y menores de 2 años y cuando son severos se pueden asociar al ALTE ${ }^{21}$.

Las infecciones urinarias se detectaron mediante exámenes de orina al igual que en el estudio de Davies ${ }^{3}$. Su origen no esta bien definido pudiendo plantearse alteraciones metabólicas no detectadas, convulsión febril manifestada por padres como ALTE o efecto de neurotoxinas en el centro respiratorio.

Se presentó sólo un trastorno metabólico, confirmando la rareza de esta etiología9 . Correspondió finalmente a una galactosemia clásica, su diagnóstico precoz permitió un tratamiento oportuno y una evolución favorable del niño.

El caso de intoxicación medicamentosa debe alertar al personal de Urgencia a preguntar acerca de la presencia de medicamentos en la casa.

Otro temor de los padres de niños que han presentado ALTE es si ellos son susceptibles al SMSL (Síndrome de Muerte Súbita del Lactante). Aproximadamente el $10 \%$ de las víctimas del SMSL habían presentado un ALTE previo horas antes de su muerte ${ }^{6}$, existiendo una cierta relación epidemiológica entre ALTE y SMSL, pero no es la regla ${ }^{16}$.

La Academia Americana de Pediatría (AAP) inició en 1992 la campaña "back to sleep", o sea la recomendación de acostar al lactante de espalda y redujo casi en un $40 \%$ la incidencia del SMSL. En cambio la prevalencia del ALTE se mantuvo sin variaciones ${ }^{22}$. En nuestra serie el $23 \%$ dormían en decúbito prono y el $20 \%$ en supino; el resto en decúbito lateral o los padres no aportaban datos.

Al alta, se instruyó a los padres sobre medidas para prevención de SMSL y maniobras de RCP; a todos los pacientes se les cita a control en un policlínico especialmente diseñado para ellos, con periodicidad preestablecida según las Guías de nuestro Servicio ${ }^{23}$, habiendo logrado implementar exámenes de mayor complejidad y un banco de monitores de apnea.

En conclusión, el médico debe tener presente la eventualidad de un ALTE en su desempeño cotidiano y frente a la diversidad etiológica se propone un protocolo de estudio por pasos que utiliza herramientas disponibles en la mayoría de los hospitales terciarios de nuestro país; mediante el cuál se lograron atribuir causas similares a las encontradas en otros estudios prospectivos, donde destaca un menor porcentaje de episodios idiopáticos. 


\section{Agradecimientos}

Agradecemos a los Doctores y Profesores: Ramiro González, (Pediatra- Neumólogo, Universidad de Chile, Hospital Exequiel González Cortés, Clínica Las Condes) y María Angélica Pérez, (Pediatra- Neumólogo, Universidad de Chile, Hospital Exequiel González Cortés, Clínica Santa María) por la revisión del trabajo. Al Dr. Darío Ramos (Jefe de Servicio de Pediatría, Hospital Guillermo Grant Benavente, Concepción) por su constante apoyo y sabios consejos. A los Pediatras de las distintas subespecialidades del Servicio de Pediatría, que en conjunto con ellos se confeccionó este protocolo. A los Médicos Pediatras, enfermeras y auxiliares de la Unidad de lactantes por su comprensión y colaboración. Finalmente a nuestros pacientes y en especial a sus madres que gracias a su preocupación y agradecimientos lograron estimular esta labor.

\section{Referencias}

1.- National Institute of Health Consensus: Development Conference on Infantile Apnea and Home Monitoring. Consensus Statement. Pediatrics 1987; 79 (2): 292300.

2.- Comité en síndrome de muerte súbita del lactante: Asociación Latinoamericana de Pediatría. Guía de práctica clínica-2005. Episodio de posible amenaza a la vida-ALTE.

3.- Davies F, Gupta R: Apparent Life threatening Events in infants presenting to an Emergency Department. Emerg Med 2002; 19; 11-16.

4.- Mitchell EA, Thompson JMD: Parental reported apnoea, admissions to hospital and sudden infant death syndrome. Acta Paediatr 1990; 417-22.

5.- Brockmann P, González X, Bertrand P, Sánchez I, Holmgren $N$ : Perfil clínico de lactantes hospitalizados por un episodio de ALTE. Rev Chil Pediatr 2006; 77 (3): 267-73.

6.- Kahn A: Recommended clinical evaluation of infants with an apparent life-threatening event. Consensus Document of the European Society for the Study and Prevention of Infant Death. Eur J Pediatr 2004; 163: 108-15.

7.- Rocca M, Jenik A, Kenny P, Agosta $G$, Ruiz AL, Gianantonio C: Eventos de aparente amenaza a la vida. Experiencia de un enfoque pediátrico interdisci- plinario. Arch Arg Pediatr 1995; 93: 85-91.

8.- Samuels $M$, Poets $C$, Noyes J, Hartmann H, Hervenston $J$, Southall D: Diagnosis and management after ALTE in infants and young children who received cardiopulmonary resuscitation. BMJ 1993; 306: 489-92.

9.- Kahn A, Grosswasser J, Scottiaux M, Rubuffat E, Franco $P$ : Clinical problems in relation to apparent lifethreatening events in infants. Acta Paediatr 1993; Suppl 389: 107-10.

10.- Rahilly $P$ : The pneumographic and medical investigation of infants suffering from apparent lifethreatening events. J Paediatr Child Health 1991; 27: 349-53.

11.- Veereman-Waters G, Bochner A, Van Caillie M: Gastroesophageal reflux in infants with a near-miss Sudden Infant Death Syndrome. J Pediatr Gastroenterol Nutr 1991; 12 (3): 319-23.

12.- Mc Carthy C, Breese C: Respiratory Syncitial Virus. Concerns and Control. Ped Rev 2003; 24 (9): 301-8.

13.- Rayyan M, Naulaers $G$, Daniels $H$, Allegaert $K$, Debeer $A$, Devlieger $H$ : Characteristics of respiratory virusrelated apnoea in three infants. Acta Paediatr 2004; 93 (6): $847-9$

14.- Southall $D$, Thomas $M$, Lambert $H$ : Severe hypoxemia in infants with pertussis. Arch Dis Child 1988; 63: 598-605.

15.- Poets, Stebbens V, Alexander S, Arrowsmith W, Salfield $S$, Southall D: Hypoxemia in infants with respiratory tract infections. Acta Paediatr 1992; 812: 536-41.

16.- Zenteno D: Eventos de aparente amenaza a la vida (ALTE): Conceptos, etiología y manejo. Neumología Pediátrica 2006; 1 (3): 133-8.

17.- Wenzel T, Schenke S, Peschegens T: Association of apnea and gastroesophageal reflux in infants investigated with the Intraluminal Impedance Technique. Pediatric Pulmonol 2001; 32: 144-8.

18.- Mousa H, Woodley FW, Metheney M, Hayes J: Testing the association between gastroesophageal reflux and apnea in infants. J Pediatr Gastroenterol Nutr 2005; 41 (2): 169-77.

19.- Byard R, Burnell R: Apparent life-threatening events and infant holding practices. Arch Dis Child 1995; 73 (6): 502-4.

20.- Brown D, Godman M: Life-threatening "epilepsy". Arch Dis Child 1991; 66: 986-87.

21.- Arens A, Gozal P, Williams J, Davidsons, Keens T: Recurrent ALTE during infancy. A manifestation of inborn errors of metabolism. J Pediatr 1993; 123: 415-8.

22.- Gersham W, Besch N, Franciosa R: A comparison of ALTE before and after the "back to sleep" campaign. WMJ 2002; 101 (1): 39-45.

23.- Guías de manejo de los Eventos de Aparente Amenaza a la Vida (ALTE): Unidad de Lactantes, Servicio de Pediatría de Hospital Guillermo Grant Benavente Concepción, Junio, 2003. 